\title{
Asymptotic formulas for the Lyapunov spectrum of fully developed shell model turbulence
}

\author{
Michio Yamada \\ Graduate School of Mathematical Sciences, University of Tokyo, Tokyo 153-8914, Japan \\ Koji Ohkitani* \\ Faculty of Integrated Arts and Sciences, Hiroshima University, Higashi-Hiroshima 739, Japan
}

(Received 30 October 1996; revised manuscript received 19 September 1997)

\begin{abstract}
The scaling behavior of the Lyapunov spectrum of a chaotic shell model for three-dimensional turbulence is studied in detail. First, we characterize the localization property of the Lyapunov vectors in wave-number space by using numerical results. By combining this localization property with Kolmogorov's dimensional argument, we deduce explicitly the asymptotic scaling law for the Lyapunov spectrum, which in turn is shown to agree well with the numerical results. This shell model is an example of high-dimensional chaotic systems for which an asymptotic scaling law is obtained for the Lyapunov spectrum. Implications of the present results for the Navier-Stokes turbulence are discussed. In particular, we conjecture that the distribution of Lyapunov exponents is not singular at null exponent. [S1063-651X(98)50206-1]
\end{abstract}

PACS number(s): 47.27.Jv, 05.45.+b, 47.52.+i

One of the most fundamental characterization of strange attractors is given by the spectrum of Lyapunov exponents, as it reflects instability of the trajectory in phase space. However, some problems regarding the Lyapunov spectrum of turbulence are left unanswered [1]. For example, in conventional Fourier representation we can characterize fully developed turbulence by the inertial subrange extending with increasing Reynolds numbers. But how can we characterize it in terms of the Lyapunov spectrum, or what property does the Lyapunov spectrum have in fluid turbulence? A simpler problem is to determine how the maximum Lyapunov exponent depends on the Reynolds number $R(=1 / \nu)$, where $\nu$ is kinematic viscosity. This problem has been studied within some shell models and it was found that the maximum Lyapunov exponent is proportional to the reciprocal of the time scale associated with the smallest eddies. The result that the time scale of the smallest eddies is responsible to the maximum Lyapunov exponent is dimensionally consistent but physically counterintuitive, because the smallest eddies are believed to be characteristic of the dissipative structures.

In this Rapid Communication we focus our attention on a shell model [2] for three-dimensional (3D) turbulence and we investigated the scaling properties of its Lyapunov spectrum in more detail than before $[3,4]$ to discuss the abovementioned issues. In this chaotic model, a set of complex variables $u_{j},(j=1,2, \ldots, N)$, which represents the velocity in the shell $k_{j}=k_{0} 2^{j}\left(k_{0}=2^{-4}\right)$, is governed by the following equations of motion:

$$
\begin{aligned}
\left(\frac{d}{d t}+\nu k_{j}^{2}\right) u_{j}= & i\left[a_{j} u_{j+1} u_{j+2}+b_{j} u_{j-1} u_{j+1}+c_{j} u_{j-1} u_{j-2}\right]^{*} \\
& +f \delta_{j, 4} .
\end{aligned}
$$

Here, * denotes complex conjugate and the coupling constants of the nonlinear terms are assumed as $a_{j}=k_{j}, b_{j}=$

\footnotetext{
*Present address: Research Institute for Mathematical Sciences, Kyoto University, Kyoto 606-8502, Japan.
}

$-\frac{1}{2} k_{j-1}, c_{j}=-\frac{1}{2} k_{j-2}$ and $b_{1}=c_{1}=c_{2}=a_{N-1}=a_{N}=b_{N}=0$ to ensure energy conservation.

A number of remarkable properties were revealed by extensive numerical and analytical studies on this model. In particular, for large Reynolds numbers solutions to this model are generally chaotic and that their time-averaged energy spectrum satisfies the same scaling law as Kolmogorov's for realistic turbulence $[3,4]$. Other aspects of this model have been also discussed, such as the intermittency effects, the probability distributions of the velocity variables [5-8], the effects of extra helicitylike invariant $[9,10]$ and the stability problem of steady solutions $[11,12]$.

So far, little is known about the structure of strange attractors associated with fully developed turbulence, and only some conjectures have been given on the nature of the Lyapunov spectrum. In the shell model it has been found that the distribution function of the Lyapunov exponents $\lambda$ appears to diverge at $\lambda=0$ in the limit of large Reynolds number [3]. The possibility of divergence in the case of NavierStokes turbulence was also pointed out before in [1] by an eigenvalue calculation of the Schrödinger operator with the use of the $\beta$ model [13]. This suggested that the inertial subrange was connected with a large number of (almost) null exponents, and indeed, a correlation between the Fourier and the Lyapunov indices was observed in the long-time average of the squared components of Lyapunov vectors $[4,14]$. However, neither its relation to the Kolmogorov scaling nor the mechanism of accumulation of null exponents was explained so far. In this Rapid Communication, we will see that how the characteristic Lyapunov spectrum is related to Kolmogorov's scaling through the localization of the Lyapunov vectors in wave number space and thereby obtain an asymptotic formula for the Lyapunov exponents.

Equations (1) are integrated numerically by the fourthorder Runge-Kutta method, together with $2 N$ linearized equations. Numerical parameters $N=19,22,24,27$ are used for four different values of viscosity $\nu$ $=10^{-6}, 10^{-7}, 10^{-8}, 10^{-9}$, respectively. The forcing is fixed as $f=5 \times 10^{-3}(1+i)$. The time step used is $\Delta t=5 \times 10^{-5}$ 


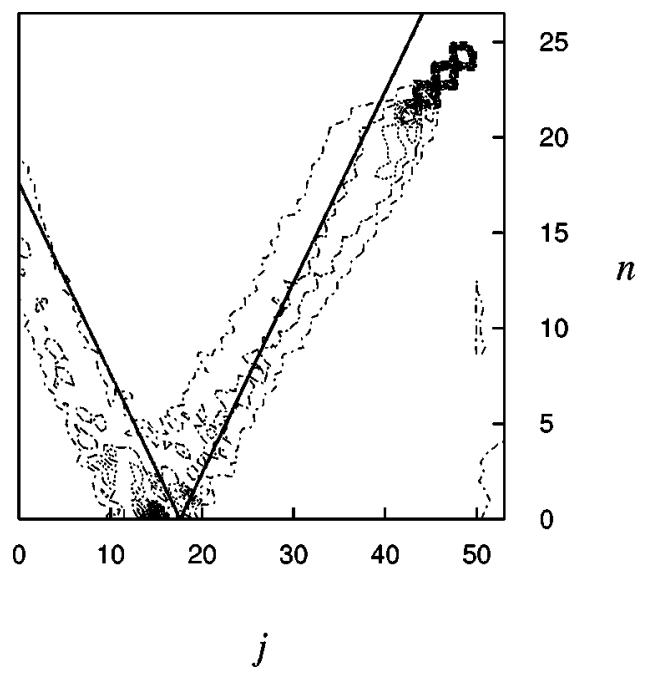

FIG. 1. Time average of squared components of the Lyapunov vectors: $\left\langle\left|v_{n}^{(j)}\right|^{2}\right\rangle$. Contour levels are $0.0489 i(i=1,2, \ldots, 10)$. Two straight lines represent the correspondence assumed in (a) and (b) of the hypotheses (2).

for $N=27$. After a transient stage, the solution apparently reaches a statistically stationary state. Below we will consider the long-time averages in this state. The energy spectrum shows six decades of the inertial subrange for $\nu$ $=10^{-9}$. The Lyapunov dimensions are estimated as $D$ $=19.8,25.4,30.2,35.0$ respectively for $N=19,22,24,27$.

Let $v_{n}^{(j)}$ be the $n$th Fourier mode of the $j$ th Lyapunov vector $(j=1,2, \ldots, 2 N, n=1,2, \ldots, N)$. We plot in Fig. 1 the squared components of the Lyapunov vectors in its time average for $N=27$

$$
E^{(j)}\left(k_{n}\right)=\left\langle\left|v_{n}^{(j)}\right|^{2}\right\rangle
$$

where the brackets denote the time average and each Lyapunov vector is normalized so that $\Sigma_{n}\left|v_{n}^{(j)}\right|^{2}=1$ for each $j$. Several distinct features are noted from the numerical results:

(i) Each Lyapunov vector has a support localized around a wave number.

(ii) The center of the support of the Lyapunov vector lies at $n \approx D / 2$ for the largest Lyapunov exponent $(j=1)$.

(iii) The central wave number of the support decreases with $j$, until the wave number reaches $n \approx 0$. The corresponding Lyapunov exponents are positive but those corresponding to $n \approx 0$ are small.

(iv) For larger $j$, the central wave number increases again, reaching $n \approx D / 2$ at $j \approx D$. The Lyapunov exponents for these are negative.

(v) For even larger $j$, the central wave number increases beyond $n \geqslant D / 2$. These Lyapunov exponents asymptotically agree with $-\nu k_{j}^{2}$; that is, the reciprocal of the viscous time scale of Eq. (1).

To summarize, for each $n(0 \leqslant n \leqslant D / 2)$ there are two Lyapunov vectors, one corresponding to a positive exponent and the other a negative one. All these features are consistent with the fact that the Lyapunov dimension measures the number of modes below the dissipation wave number and that there are two degrees of freedom for each wave number, the real and the imaginary parts of the velocity variables.
On the basis of the above observations we will introduce the following set of hypotheses regarding the Lyapunov vectors in the inertial subrange $j \leqslant D$ for $D \gg 1$ :

(1) Lyapunov exponents are positive for $1 \leqslant j \leqslant D / 2$ and negative for $j>D / 2$.

(2) Each Lyapunov vector in wave number space is localized around a wave number. Let $k_{n_{j}}=k_{0} 2^{n_{j}}$ be the localized wave number for $j$ th Lyapunov vector, then $n_{j}$ is given by

(a) $n_{j}=D / 2-j+1$ for $1 \leqslant j \leqslant D / 2$,

(b) $n_{j}=j-D / 2$ for $D / 2+1 \leqslant j \leqslant D$.

(3) In the inertial subrange, the $j$ th Lyapunov exponent $(j \leqslant D)$ is inversely proportional to the Kolmogorov's time scale $\epsilon^{-1 / 3} k_{n_{j}}^{-2 / 3}$.

The last hypothesis, a combination of the Kolmogorov's dimensional argument and the localization of the Lyapunov vectors in wave number space, is equivalent to assume that the Lyapunov exponents can be represented in terms of the energy dissipation rate $\epsilon$ and wave number $k$ at which the Lyapunov vectors are localized. From these hypotheses, we deduce the following formulas for the Lyapunov exponents

$$
\lambda_{j} \sim\left\{\begin{array}{l}
\epsilon^{1 / 3} k_{n_{j}}^{2 / 3}=\epsilon^{1 / 3}\left(2 k_{0}\right)^{2 / 3} 2^{(D-2 j) / 3} \\
\text { for } 1 \leqslant j \leqslant D / 2, \\
-\epsilon^{1 / 3} k_{n_{j}}^{2 / 3}=-\epsilon^{1 / 3}\left(k_{0}\right)^{2 / 3} 2^{(2 j-D) / 3} \\
\text { for } D / 2+1 \leqslant j \lesssim D .
\end{array}\right.
$$

We now rescale the Lyapunov exponent as $\lambda_{j} / H$, where $H=\Sigma_{\lambda_{j}>0} \lambda_{j}$ is the Kolmogorov-Sinai entropy. This is equivalent to choose time $\tau=H t$ such that $H$ is normalized as unity. It should be remarked that this choice of time neither influences the geometric structure of the strange attractors nor the invariant measure on them. Noting that

$$
H=\sum_{j=1}^{D / 2} \lambda_{j} \sim \epsilon^{1 / 3}\left(2 k_{0}\right)^{2 / 3} \frac{2^{D / 3}-1}{2^{2 / 3}-1}
$$

we conclude that

$$
\frac{\lambda_{j}}{H}=\left\{\begin{array}{cc}
\left(2^{2 / 3}-1\right) \frac{2^{D / 3}}{2^{D / 3}-1} 2^{-2 j / 3} & \text { for } 1 \leqslant j \leqslant D / 2, \\
-\frac{2^{2(j-D / 2) / 3}\left(2^{2 / 3}-1\right)}{2^{D / 3}-1} & \text { for } D / 2+1 \leqslant j \lesssim D .
\end{array}\right.
$$

Because these expressions are free from arbitrary parameters, we can compare this phenomenological theory with the results of numerical simulations. In Fig. 2 we plot the positive Lyapunov exponents $\lambda_{j} / H$ against $j$ together with the theoretical curve for $D \gg 1$

$$
\frac{\lambda_{j}}{H}=\left(2^{2 / 3}-1\right) 2^{-2 j / 3},
$$

which can be obtained from Eq. (5). The numerical results and the phenomenological theory agree well generally and a better agreement between them is obtained for larger $N$, that 


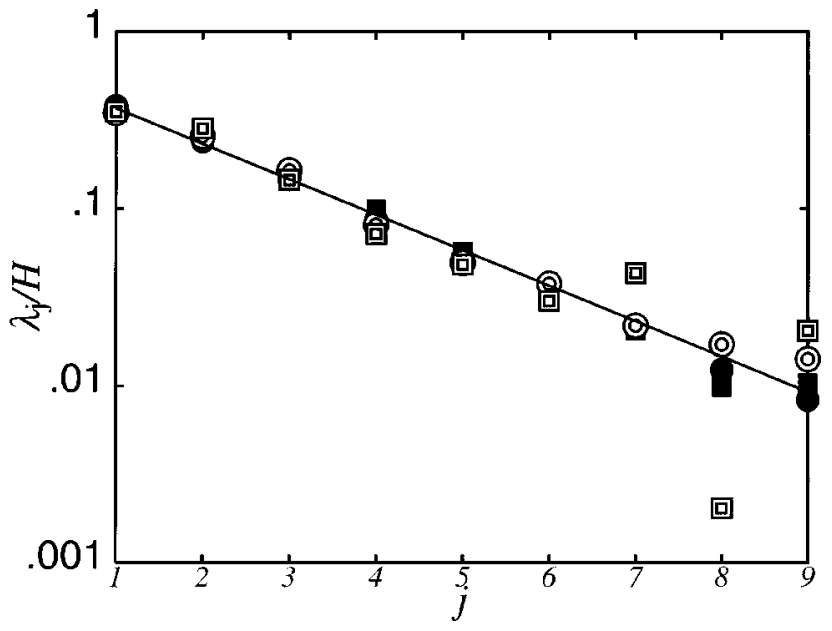

FIG. 2. Distribution of the Lyapunov exponents $\lambda_{j} / H$ : circles $(N=19)$, squares $(N=22)$, closed squares $(N=24)$, and closed circles $(N=27)$. The dashed line denotes the theoretical prediction: $\lambda_{j} / H=\left(2^{2 / 3}-1\right) 2^{-2 j / 3}$.

is, for larger Lyapunov dimensions. In Fig. 3, we also plot $\sum_{i=1}^{j} \lambda_{i} / H$ and compare it with the theoretical prediction

$$
\sum_{i=1}^{j} \frac{\lambda_{i}}{H}=1-2^{-2 j / 3}
$$

Again, the theoretical prediction agrees well with the numerical data and this agreement is better for larger $N$ [15]. For $j>D / 2$, on the other hand, only a rough agreement is seen between the theory and the numerical results, but at present we do not know why the agreement is less clear.

The agreement between Eq. (7) and numerical results support validity of the working hypotheses introduced above to derive Eq. (5). In particular, this shows that each wave number in the inertial subrange corresponds to two Lyapunov vectors, one is stable and the other unstable, adding more

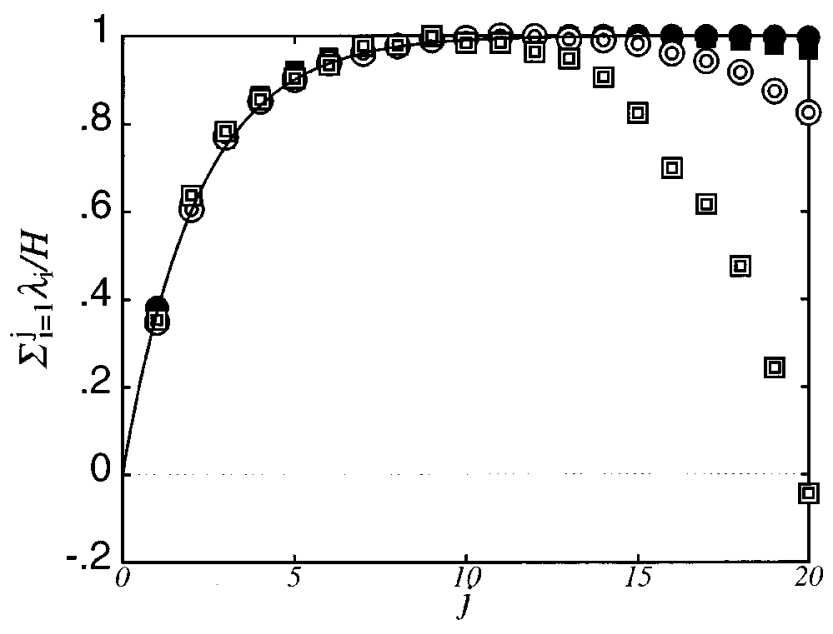

FIG. 3. Cumulated distribution of the Lyapunov exponents $\sum_{i=1}^{j} \lambda_{i} / H$. Symbols are the same as in Fig. 2. The dashed line denotes the theoretical prediction: $\sum_{i=1}^{j} \lambda_{i} / H=1-2^{-2 j / 3}$. support to the dual structure of the Lyapunov vectors observed previously [4]. The dual structure explains at least partially why the dissipative structures appear to be relevant to the maximum Lyapunov exponent; the largest wave number mode of the Lyapunov vectors in the inertial subrange is characteristic of both the most stable and unstable motions. We note that the localization of Lyapunov vectors in the disspation range was observed in [4,5] during the burst-like intermittent energy transfer. These bursts correspond to one branch of the V-shaped correlation observed in the long-time average of the Lyapunov vectors.

Now we would like to consider what is predicted for realistic turbulence by assuming the localization of the Lyapunov vectors. Suppose that the support of the Lyapunov vectors for the Navier-Stokes turbulence with high Reynolds number is localized in wave number space as in the case of the shell model. This implies that the Lyapunov exponents are given by the reciprocal of the relevant Kolmogorov time scale $\lambda \sim \epsilon^{1 / 3} k^{2 / 3}$, where $k$ is the wave number associated with the Lyapunov vector [16]. The number of Lyapunov exponents which lie in $[\lambda, \lambda+d \lambda]$ is then proportional to $4 \pi k^{2} d k$. Thus, the density distribution function of Lyapunov exponents $P(\lambda)$ satisfies $P(\lambda) d \lambda \sim 4 \pi k^{2} d k$, which leads to

$$
P(\lambda) \sim \lambda^{7 / 2}
$$

It should be noted that this distribution $P(\lambda)$ does not diverge at $\lambda=0$, in a marked contrast to the case of the shell model, where $P(\lambda)$ has a singularity $1 / \lambda$ at $\lambda=0$. In retrospect, this divergence comes from the condensation of modes at null wave number, which is attributed to an artifact of the octave-discretization of wave numbers. As noted above, the divergence was conjectured by Ruelle for the Navier-Stokes turbulence [1], but Ruelle's argument employed the $\beta$ model [13], in which the wave numbers are also discretized in octaves. We also note that the formula (9) is consistent with results of Wang and Gaspard [17] for Kolmogorov-Sinai entropy and Aurell et al. for the maximum Lyapunov exponent [18].

Here comparison to other theories on the properties of the Lyapunov spectrum may be in order. First, it was shown in [19] that for a class of dissipative dynamical systems with a simple dissipation term the Lyapunov spectrum is symmetric with respect to a constant that depends on the dissipation of the system. Unfortunately the shell model does not belong to this class because its dissipation term depends on the independent variables (wave numbers). Still, a pairing phenomenon of positive and negative exponents was predicted from a nonequilibrium statistical mechanical formulation of turbulence [20]. Also in the shell model the paring of the Lyapunov exponents are clearly observed, especially in the inertial subrange where the dissipation is considered to be very small. Further investigation would be of interest on the basis of this formulation. Apart from dissipative turbulent systems, we note that another approach to the property of the Lyapunov spectrum was given by Bohr, Grinstein, and Jayaprakash [21] for a conservative system without cascade 
process. They discussed a class of coupled maps (see also $[22,23])$ with a particular choice of couplings, which permits an analytical derivation of $P(\lambda) \propto \lambda^{-1 / 2}$.

In the present paper, we have shown that the localization of the Lyapunov vectors permits us to relate the Lyapunov exponents to some conventional statistical quantities. The lo- calization of the Lyapunov vectors are strongly related with the scaling property in the present system, and we conjecture that the localization may be rather universal among a class of high-dimensional chaotic systems with a scale invariance. Further study on the lo-calization of the Lyapunov vectors will be reported elsewhere.
[1] D. Ruelle, Commun. Math. Phys. 87, 287 (1982).

[2] E. B. Gledzer, Sov. Phys. Dokl. 18, 216 (1973).

[3] M. Yamada and K. Ohkitani, J. Phys. Soc. Jpn. 56, 4210 (1987).

[4] K. Ohkitani and M. Yamada, Prog. Theor. Phys. 81, 329 (1989).

[5] M. H. Jensen, D. Paladin, and A. Vulpiani, Phys. Rev. A 43, 798 (1991).

[6] D. Pisarenko, L. Biferale, D. Courvoisier, U. Frisch, and M. Vergassola, Phys. Fluids A 5, 2533 (1993).

[7] R. Benzi, L. Biferale, and G. Parisi, Physica D 65, 163 (1993).

[8] M. Yamada,, S. Kida and K. Ohkitani, Unstable and Turbulent Motion of Fluid, edited by S. Kida (World Scientific, Singapore, 1993), pp. 188-199.

[9] L. Kadanoff, D. Lohse, J. Wang, and R. Benzi, Phys. Fluids 7, 617 (1995).

[10] L. Biferale, A. Lambert, R. Lima, and G. Paladin, Physica D 80, 105 (1995).

[11] L. Biferale, M. Blank, and U. Frisch, J. Stat. Phys. 75, 781 (1994).

[12] L. Kadanoff, D. Lohse, and N. Schöerghofer, Physica D 100, 165 (1997).
[13] U. Frisch, P.L. Sulem, and M. Nelkin, J. Fluid Mech. 87, 719 (1978).

[14] M. Yamada and K. Ohkitani, Prog. Theor. Phys. 79, 1265 (1987).

[15] In a previous paper [3] we conjectured from the numerical fittings of the simulation data that there might be a viscosityindependent relation between $\sum_{i=1}^{j} \lambda_{i} / H$ and $j / D$. But the present phenomenological argument and its confirmation by numerical simulations at larger $N$ show that the relation should be corrected as Eq. (7).

[16] For simplicity, we consider a Navier-Stokes flow under periodic boundary conditions in a box of size $L^{3}$.

[17] X.-J. Wang and P. Gaspard, Phys. Rev. A 46, R3000 (1992).

[18] E. Aurell, G. Beffetta, A. Crisanti, G. Paladin, and A. Vulpiani, Phys. Rev. E 53, 2337 (1996).

[19] U. Dressler, Phys. Rev. A 38, 2103 (1988).

[20] G. Gallavotti and E. G. D. Cohen, Phys. Rev. Lett. 74, 2694 (1995); G. Gallavotti, Physica D 105, 163 (1997).

[21] T. Bohr, G. Grinstein, and C. Jayapraksh, Chaos 5, 412 (1995).

[22] R. O. Grigoriev and M. C. Cross, e-print chao-dyn/9702001.

[23] H. Chaté, Europhys. Lett. 21, 419 (1993). 